Proc. Indian Acad. Sci. (Math. Sci.) Vol. 116, No. 2, May 2003, pp. 121-136.

Printed in India

\title{
The abstruse meets the applicable: Some aspects of time-frequency analysis
}

\author{
G B FOLLAND \\ Department of Mathematics, University of Washington, Seattle, WA 98195-4350, USA \\ E-mail: folland@math.washington.edu
}

MS received 6 February 2006

\begin{abstract}
The area of Fourier analysis connected to signal processing theory has undergone a rapid development in the last two decades. The aspect of this development that has received the most publicity is the theory of wavelets and their relatives, which involves expansions in terms of sets of functions generated from a single function by translations and dilations. However, there has also been much progress in the related area known as time-frequency analysis or Gabor analysis, which involves expansions in terms of sets of functions generated from a single function by translations and modulations. In this area there are some questions of a concrete and practical nature whose study reveals connections with aspects of harmonic and functional analysis that were previously considered quite pure and perhaps rather exotic. In this expository paper, I give a survey of some of these interactions between the abstruse and the applicable. It is based on the thematic lectures which I gave at the Ninth Discussion Meeting on Harmonic Analysis at the Harish-Chandra Research Institute in Allahabad in October 2005.
\end{abstract}

Keywords. Gabor frames; time-frequency analysis; discrete Heisenburg group.

\section{Background and statements of theorems}

In this section we develop some basic ideas of time-frequency analysis and lead up to the main results to be discussed in this paper. For a more comprehensive account of this subject we recommend the book of Gröchenig [14]; see also Daubechies [5].

For an $L^{2}$ function $f$ on $\mathbb{R}^{d}$, the Fourier transform and its inversion formula

$$
\hat{f}(\omega)=\int f(t) \mathrm{e}^{-2 \pi i \omega \cdot t} \mathrm{~d} t, \quad f(t)=\int \hat{f}(\omega) \mathrm{e}^{2 \pi i \omega \cdot t} \mathrm{~d} \omega
$$

provide the expansion of $f$ in terms of the pure 'sine waves' $\mathrm{e}^{2 \pi i \omega \cdot t}$. (When $d=1$ it is often appropriate to refer to $t$ as 'time' and $\omega$ as 'frequency.' We shall sometimes use these terms even when dealing with general $d$, for which the basic mathematical structure is exactly the same.) The Fourier transform is a marvelous tool, but since it involves the whole function $f$ at once, it is not an efficient way to analyze the ways in which different frequencies enter into $f$ at different times (as is of paramount importance, for example, in music).

One way to produce a 'local' Fourier analysis of a function $f$ is to use the windowed Fourier transform, which is also known as the short-time Fourier transform and is closely related to the cross-ambiguity function of radar theory and the Fourier-Wigner transform in [10]. The idea is simple: one fixes an $L^{2}$ function $\phi$ on $\mathbb{R}^{d}$ such that $\|\phi\|_{2}=1$ and 
considers the Fourier transform not of $f$ but of $f$ multiplied by translates of $\phi$, obtaining a function $V_{\phi} f$ on $\mathbb{R}^{d} \times \mathbb{R}^{d}$ :

$$
V_{\phi} f(\omega, x)=\int f(t) \overline{\phi(t-x)} \mathrm{e}^{-2 \pi i \omega \cdot t} \mathrm{~d} t .
$$

$V_{\phi} f$ is called the windowed Fourier transform of $f$ with window $\phi$. (The reasons for the complex conjugation on $\phi$ and the normalization $\|\phi\|_{2}=1$ will become apparent shortly.) If one takes $\phi$ to be a nonnegative function supported on an interval $I$ centered at the origin (or at least negligibly small outside $I$ ), then $V_{\phi} f(\omega, x)$ measures how much the frequency $\omega$ contributes to the portion of $f$ that lives on the interval $I+x$ centered at $x$.

To obtain the inversion formula for the operator $V_{\phi}$, we observe that the map $(\phi, f) \mapsto V_{\phi} f$ is the restriction to functions of the form $F(x, t)=\overline{\phi(x)} f(t)$ of the linear map $\tilde{V}: L^{2}\left(\mathbb{R}^{2 d}\right) \rightarrow L^{2}\left(\mathbb{R}^{2 d}\right)$ defined by

$$
\tilde{V} F(\omega, x)=\int F(t-x, t) \mathrm{e}^{-2 \pi i \omega \cdot t} \mathrm{~d} t .
$$

$\tilde{V}$ is the composition of the measure-preserving change of variable $(x, t) \mapsto(t-x, t)$ with the Fourier transform in the second variable, so it is unitary on $L^{2}$. It follows that the windowed Fourier transform is an isometry from $L^{2}\left(\mathbb{R}^{d}\right)$ into $L^{2}\left(\mathbb{R}^{2 d}\right)$ :

$$
\left\|V_{\phi} f\right\|_{2}=\|\phi\|_{2}\|f\|_{2}=\|f\|_{2} .
$$

Therefore, we have $V_{\phi}^{*} V_{\phi}=I$, and an easy calculation of $V_{\phi}^{*}$ then yields the inversion formula

$$
f(t)=\iint V_{\phi} f(\omega, x) \phi(t-x) \mathrm{e}^{2 \pi i \omega \cdot t} \mathrm{~d} x \mathrm{~d} \omega .
$$

(As with the ordinary Fourier transform, this integral is absolutely convergent only for $f$ in a dense subspace of $L^{2}$ and must be interpreted by a limiting process in general.)

Let us look at this from another viewpoint. For $\omega, x \in \mathbb{R}^{d}$ we introduce the modulation operator $M_{\omega}$ and the translation operator $T_{x}$ by

$$
M_{\omega} f(t)=\mathrm{e}^{2 \pi i \omega \cdot t} f(t), \quad T_{x} f(t)=f(t-x) .
$$

Thus

$$
\begin{aligned}
& M_{\omega} T_{x} f(t)=\mathrm{e}^{2 \pi i \omega \cdot t} f(t-x), \\
& T_{x} M_{\omega} f(t)=\mathrm{e}^{2 \pi i \omega \cdot(t-x)} f(t-x)=\mathrm{e}^{-2 \pi i \omega \cdot x} M_{\omega} T_{x} f(t),
\end{aligned}
$$

and (11) and (2) become

$$
V_{\phi} f(\omega, x)=\left\langle f, M_{\omega} T_{x} \phi\right\rangle, \quad f=\iint V_{\phi} f(\omega, x) M_{\omega} T_{x} \phi \mathrm{d} x \mathrm{~d} \omega .
$$

That is, we are using the time-frequency translates (i.e., translates and modulates) of $\phi$, $M_{\omega} T_{x} \phi$, as a 'basic set' of functions by means of which one can express an arbitrary $L^{2}$ function $f$.

However, the set $\left\{M_{\omega} T_{x} \phi: \omega, x \in \mathbb{R}^{d}\right\}$ is highly overcomplete, and in general one can expand an arbitrary $f$ using only a suitable discrete subset of it. For example, if $\phi$ is the 
characteristic function of the unit cube $[0,1]^{d}$, the set $\left\{M_{j} T_{k} \phi: j, k \in \mathbb{Z}^{d}\right\}$ is actually an orthonormal basis for $L^{2}\left(\mathbb{R}^{d}\right)$. (It is the basis one obtains by tiling $\mathbb{R}^{d}$ by cubes of unit side length with vertices in the integer lattice and using the usual Fourier basis on each cube.) Such discrete 'basic sets' have an obvious advantage from a computational point of view, as integrals must be approximated by discrete sums for numerical work anyway. We are thus led to the following definition.

Given $\phi \in L^{2}\left(\mathbb{R}^{d}\right)$ and $\alpha, \beta>0$, let

$$
\mathcal{G}(\phi, \alpha, \beta)=\left\{M_{\alpha j} T_{\beta k} \phi: j, k \in \mathbb{Z}^{d}\right\} .
$$

$\mathcal{G}(\phi, \alpha, \beta)$ is called the Gabor system determined by $(\phi, \alpha, \beta)$. (The name is in honor of the electrical engineer D Gabor, who suggested in his ground-breaking paper of 1946 [13] that $\mathcal{G}\left(\mathrm{e}^{-\pi t^{2} / \alpha^{2}}, 1 / \alpha, \alpha\right)$ should be a useful 'basic set'. He was not entirely correct - see $\S 3.4$ of [10] - but the essential idea was still a good one.) Evidently this concept can be generalized: one can consider $\left\{M_{\omega} T_{x} \phi:(\omega, x) \in \Lambda\right\}$ where $\Lambda$ is a more general discrete subset of $\mathbb{R}^{2 d}$. We shall consider such generalizations later, but we stick with the lattice $\Lambda=\alpha \mathbb{Z}^{d} \times \beta \mathbb{Z}^{d}$ for now.

The first question that must be addressed is the following: For which $\phi, \alpha$, and $\beta$ does $\mathcal{G}(\phi, \alpha, \beta) \operatorname{span} L^{2}\left(\mathbb{R}^{d}\right)$ ? (By 'span' we mean that its finite linear span is dense in $L^{2}\left(\mathbb{R}^{d}\right)$.) This question, as it stands, is too broad to admit a reasonable answer. In particular, if one is given $\phi$, the set of $(\alpha, \beta)$ for which $\mathcal{G}(\phi, \alpha, \beta)$ spans $L^{2}$ depends strongly on $\phi$. For example, if $d=1$ and $\phi$ (resp. $\hat{\phi}$ ) is supported in an interval of length $l$, for $\mathcal{G}(\phi, \alpha, \beta)$ to span $L^{2}$ it is obviously necessary that $\beta \leq l$ (resp. $\alpha \leq l$ ). On the other hand, no matter what $\phi$ is, it is always necessary that the lattice $\alpha \mathbb{Z}^{d} \times \beta \mathbb{Z}^{d}$ should be sufficiently dense in $\mathbb{R}^{d} \times \mathbb{R}^{d}$. Indeed, we have the following:

Theorem 1. If $\alpha \beta>1$, there is no $\phi \in L^{2}$ such that $\mathcal{G}(\phi, \alpha, \beta)$ spans $L^{2}\left(\mathbb{R}^{d}\right)$.

When $\alpha \beta>1$ and $\alpha \beta$ is rational, Daubechies [4] has shown how to produce, for any given $\phi$, an explicit $f \neq 0$ such that $f \perp \mathcal{G}(\phi, \alpha, \beta)$. (See also p. 107 of [5] for the very easy case where $\alpha=1, \beta=2$.) However, when $\alpha \beta$ is irrational, a deeper argument is necessary. The reason for this rather surprising distinction lies in the structure of the group of operators $G_{\alpha, \beta}$ generated by the modulations and translations $M_{\alpha j}$ and $T_{\beta k}\left(j, k \in \mathbb{Z}^{d}\right)$. In view of (3), we have

$$
G_{\alpha, \beta}=\left\{\mathrm{e}^{2 \pi i \alpha \beta l} M_{\alpha j} T_{\beta k}: j, k \in \mathbb{Z}^{d}, l \in \mathbb{Z}\right\} .
$$

This group is a homomorphic image of the discrete Heisenberg group $\mathbb{H}_{d}$ whose underlying set is $\mathbb{Z}^{d} \times \mathbb{Z}^{d} \times \mathbb{Z}$ and whose group law is

$$
(j, k, l) \cdot\left(j^{\prime}, k^{\prime}, l^{\prime}\right)=\left(j+j^{\prime}, k+k^{\prime}, l+l^{\prime}+k \cdot j^{\prime}\right) .
$$

(This is often written with the roles of $j$ and $k$ switched.) Indeed, the map

$$
\pi_{\alpha, \beta}(j, k, l)=\mathrm{e}^{-2 \pi i \alpha \beta l} M_{\alpha j} T_{\beta k}
$$

is easily seen to be a unitary representation of $\mathbb{H}_{d}$ whose image is $G_{\alpha, \beta}$. When $\alpha \beta$ is irrational, $\pi_{\alpha, \beta}$ is an isomorphism of groups. On the other hand, when $\alpha \beta$ is rational, say $\alpha \beta=p / q$ in lowest terms, we have

$$
\operatorname{ker}\left(\pi_{\alpha, \beta}\right)=q Z \equiv\{(0,0, q l): l \in \mathbb{Z}\}
$$


and hence

$$
G_{\alpha, \beta} \cong \mathbb{H}_{d} / q Z \cong\left\{(j, k, l): j, k \in \mathbb{Z}^{d}, l \in \mathbb{Z} / q \mathbb{Z}\right\},
$$

the group law again being given by 6 with addition $\bmod q$ in the last coordinate. Now, observe that $Z=\{(0,0, l): l \in \mathbb{Z}\}$ is both the center and the commutator subgroup of $\mathbb{H}_{d}$. The group $\mathbb{H}_{d} / q Z$ is 'almost Abelian': its commutator subgroup is finite, and it has normal Abelian subgroups of finite index (for example, $\left\{(j, q k, l): j, k \in \mathbb{Z}^{d}, l \in \mathbb{Z} / q \mathbb{Z}\right\}$ ). As a result, the harmonic analysis of $\mathbb{H}_{d} / q Z$ can be reduced to Abelian harmonic analysis. But $\mathbb{H}_{d}$ itself is a discrete group that is not 'almost Abelian,' and consequently it is not even type I. This means that its harmonic analysis exhibits various pathologies and involves queer beasts such as von Neumann factors of type II (see Chapter 7 of [11] for a fuller explanation of these matters).

In $\S 2$ we develop the basic properties of the von Neumann algebra generated by $G_{\alpha, \beta}$, and in $\S 3$ we discuss several proofs of Theorem 1.

We now turn to a different question. Suppose that $\mathcal{G}(\phi, \alpha, \beta)$ does span $L^{2}$; can we use it in an efficient way to expand an arbitrary $f \in L^{2}$ ? The condition that allows everything to work smoothly is that $\mathcal{G}(\phi, \alpha, \beta)$ should be a frame for $L^{2}$. The notion of frame was introduced by Duffin and Schaeffer in 1952, but it was not fully exploited until a third of a century later, when Daubechies et al [6] showed how handy frames could be in signal analysis. We make a brief detour into abstract Hilbert space theory to explain this idea; see [5] or [14] for a fuller discussion.

A bit of notation that will be employed throughout this paper: If $I$ is a discrete set, we denote functions on $I$ by lower-case boldface letters such as $\mathbf{c}$, and the value of $\mathbf{c}$ at $i \in I$ is denoted by $c_{i}$.

Let $\mathcal{H}$ be a separable Hilbert space. A countable set $\left\{e_{i}\right\}_{i \in I} \subset \mathcal{H}$ is called a frame for $\mathcal{H}$ if there exist $C_{1}, C_{2}>0$ such that for all $f \in \mathcal{H}$ we have the 'frame inequalities'

$$
C_{1}\|f\|_{2} \leq \sum_{i \in I}\left|\left\langle f, e_{i}\right\rangle\right|^{2} \leq C_{2}\|f\|_{2}
$$

The second inequality in $(9)$ means that the linear map $A$ from $\mathcal{H}$ to functions on $I$ defined by $(A f)_{i}=\left\langle f, e_{i}\right\rangle$ is bounded from $\mathcal{H}$ to $l^{2}(I)$. Its adjoint $A^{*}: l^{2}(I) \rightarrow \mathcal{H}$ is easily seen to be $A^{*} \mathbf{c}=\sum c_{i} e_{i}$, where the series converges unconditionally. The composition $S=A^{*} A: \mathcal{H} \rightarrow$ $\mathcal{H}$, given by

$$
S f=\sum\left\langle f, e_{i}\right\rangle e_{i},
$$

is called the frame operator. Since $\langle S f, f\rangle=\Sigma\left|\left\langle f, e_{i}\right\rangle\right|^{2}$, the frame inequalities (9) are equivalent to the operator inequalities $C_{1} I \leq S \leq C_{2} I$; in particular, $S$ is invertible, and its inverse satisfies $C_{2}^{-1} I \leq S^{-1} \leq C_{1}^{-1} I$. Since

$$
\sum\left|\left\langle f, S^{-1} e_{i}\right\rangle\right|^{2}=\sum\left|\left\langle S^{-1} f, e_{i}\right\rangle\right|^{2}=\left\langle S\left(S^{-1} f\right), S^{-1} f\right\rangle=\left\langle S^{-1} f, f\right\rangle,
$$

these inequalities in turn imply that $\left\{S^{-1} e_{i}\right\}_{i \in I}$ is again a frame (with frame constants $C_{2}^{-1}$ and $\left.C_{1}^{-1}\right)$; it is called the dual frame. The two frames $\left\{e_{i}\right\}$ and $\left\{S^{-1} e_{i}\right\}$ can now be used together to produce expansion formulas for a general $f \in \mathcal{H}$ in terms of either frame:

$$
\begin{aligned}
& f=S\left(S^{-1} f\right)=\sum\left\langle S^{-1} f, e_{i}\right\rangle e_{i}=\sum\left\langle f, S^{-1} e_{i}\right\rangle e_{i}, \\
& f=S^{-1}(S f)=S^{-1}\left(\sum\left\langle f, e_{i}\right\rangle e_{i}\right)=\sum\left\langle f, e_{i}\right\rangle S^{-1} e_{i} .
\end{aligned}
$$


We now return to the Gabor system $\mathcal{G}(\phi, \alpha, \beta)$. If this system is a frame, an easy calculation shows that the frame operator $S$ commutes with every $M_{\alpha j}$ and $T_{\beta k}$, so the dual frame is again a Gabor system, namely, $\mathcal{G}\left(S^{-1} \phi, \alpha, \beta\right)$; we call $S^{-1} \phi$ the dual window to $\phi$.

Incidentally, Theorem 1 implies that if there exists $\phi \in L^{2}\left(\mathbb{R}^{d}\right)$ such that $\mathcal{G}(\phi, \alpha, \beta)$ is a frame, then $\alpha \beta \leq 1$, but this result is easier to prove than Theorem 1 itself (see p. 108 of [5] or Corollary 7.5.1 of [14]).

In the applications of Gabor systems, one is generally interested in using windows with good time-frequency localization, that is, windows $\phi$ for which both $\phi$ and $\hat{\phi}$ have reasonably rapid decay at infinity, or — what is more or less the same thing — that the windowed Fourier transform $V_{\gamma} \phi(\omega, x)$, for some nice fixed window $\gamma$, has rapid decay in both $\omega$ and $x$. Experience has shown that a good quantitative measure of this decay is given by the norms that characterize the so-called modulation spaces $M_{v}^{1}$, which are defined as follows.

A subexponential weight on $\mathbb{R}^{2 d}$ is a function $v: \mathbb{R}^{2 d} \rightarrow[0, \infty)$ of the form $v(\xi)=$ $\mathrm{e}^{\sigma(\rho(\xi))}$ where $\rho$ is a seminorm on $\mathbb{R}^{2 d}$ and $\sigma$ is a nonnegative concave function on $[0, \infty)$ such that $\sigma(0)=0$ and $\lim _{r \rightarrow \infty} \sigma(r) / r=0$. (Examples: $v_{1}(\omega, x)=(1+|\omega|+|x|)^{a}$, $v_{2}(\omega, x)=(1+|\omega|)^{a}$, and $v_{3}(\omega, x)=\mathrm{e}^{|x|^{b}}$, where $a>0$ and $0<b<1$.) Such a weight is always submultiplicative: $v\left(\xi_{1}+\xi_{2}\right) \leq v\left(\xi_{1}\right) v\left(\xi_{2}\right)$. Given a subexponential weight $v$, the modulation space $M_{v}^{1}$ is defined as

$$
M_{v}^{1}=\left\{f \in L^{2}\left(\mathbb{R}^{d}\right):\|f\|_{1, v}=\int_{\mathbb{R}^{2 d}}\left|V_{\gamma} f(\xi)\right| v(\xi) \mathrm{d} \xi<\infty\right\},
$$

where $\gamma$ is some fixed Schwartz-class window; $M_{v}^{1}$ turns out to be independent of the choice of $\gamma$, as is the equivalence class of the norm $\|f\|_{1, v}$. Evidently the notion of modulation space can be generalized - for example, by using an $L^{p}$ norm rather than the $L^{1}$ norm - but $M_{v}^{1}$ will suffice for our purposes. For a detailed treatment of modulation spaces we refer to Chapter 11 of [14] and [8]. Incidentally, $M_{1}^{1}$ (i.e., $M_{v}^{1}$ where $v \equiv 1$ ) is the Feichtinger algebra, often denoted by $S_{0}\left(\mathbb{R}^{d}\right)$ (see $[8]$ ).

Now, suppose $\phi$ is a window such that $\mathcal{G}(\phi, \alpha, \beta)$ is a frame for $L^{2}\left(\mathbb{R}^{d}\right)$. If $\phi$ has good time-frequency localization in the sense that $\phi \in M_{v}^{1}$ for some suitable weight $v$, it is obviously desirable that the dual window $S^{-1} \phi$ should also belong to $M_{v}^{1}$. That this is indeed the case is the second major result we wish to discuss.

Theorem 2. Suppose $v$ is a subexponential weight on $\mathbb{R}^{2 d}$ and $\alpha, \beta>0$. If $\phi \in M_{v}^{1}$ is a window such that $\mathcal{G}(\phi, \alpha, \beta)$ is a frame, then the frame operator $S$ maps $M_{v}^{1}$ bijectively onto itself. In particular, $S^{-1} \phi \in M_{v}^{1}$.

This theorem was first proved by Feichtinger and Gröchenig [9] in the case where $\alpha \beta$ is rational. The general case is a more recent result of Gröchenig and Leinert [15]; its proof involves some abstract machinery that was not needed for the rational case. The underlying reason for this dichotomy is the same as in Theorem 1: one needs a result about a noncommutative convolution on $\mathbb{Z}^{2 d}$ that is closely related to the group $G_{\alpha, b}$ defined in (5). When $\alpha \beta$ is rational, these results can be obtained by Abelian harmonic analysis, but the general case requires a different approach.

In more detail, the crucial ingredient for the proof of Theorem 2 is a noncommutative analogue of a classic result of Norbert Wiener. Wiener's theorem is usually stated as follows: If $f$ is a continuous, nonvanishing, periodic function on $\mathbb{R}$ whose Fourier series 
is absolutely convergent, then the Fourier series of $1 / f$ is also absolutely convergent. However, by passing from $f$ to its sequence $\mathbf{c}$ of Fourier coefficients, this result can also be stated as a theorem about the convolution algebra $l^{1}(\mathbb{Z})$. Indeed, taking into account the fact that the nonvanishing of $f$ is equivalent to the invertibility of the operator $g \mapsto f g$ on $L^{2}(\mathbb{R} / \mathbb{Z})$, and hence of the operator $\mathbf{a} \mapsto \mathbf{c} * \mathbf{a}$ on $l^{2}(\mathbb{Z})$, Wiener's theorem can be restated as follows: Suppose $\mathbf{c} \in l^{1}(\mathbb{Z})$ and the map $\mathbf{a} \mapsto \mathbf{c} * \mathbf{a}$ is invertible as an operator on $l^{2}(\mathbb{Z})$. Then $\mathbf{c}$ is invertible in the convolution algebra $l^{1}(\mathbb{Z})$.

The setting for the noncommutative analogue of Wiener's theorem is as follows. Given a real number $\gamma$, we define the operation of $\gamma$-twisted convolution, denoted by $\natural_{\gamma}$, on $l^{1}\left(\mathbb{Z}^{d} \times \mathbb{Z}^{d}\right)\left(\right.$ or $l^{1}\left(\mathbb{Z}^{2 d}\right)$ for short) by

$$
\left(\mathbf{a} \natural_{\gamma} \mathbf{b}\right)_{j k}=\sum_{l, m} a_{l m} b_{(j-l)(k-m)} \mathrm{e}^{-2 \pi i \gamma(j-l) \cdot m} .
$$

We also define an involution $\mathbf{a} \mapsto \mathbf{a}^{* \gamma}$ on $l^{1}\left(\mathbb{Z}^{2 d}\right)$ by

$$
\left(\mathbf{a}^{* \gamma}\right)_{j k}=\overline{a_{(-j)(-k)}} \mathrm{e}^{-2 \pi i \gamma j \cdot k} .
$$

(Note: these definitions differ from the ones in [15] by the minus signs in the exponents. This is to compensate for the fact that in [15], time-frequency shifts are written as $T_{\alpha j} M_{\beta k}$ rather than $M_{\alpha j} T_{\beta k}$.) $l^{1}\left(\mathbb{Z}^{2 d}\right)$ is a Banach $*$-algebra with product $\natural_{\gamma}$ and involution $*_{\gamma}$. We shall denote this algebra by $\mathcal{A}_{\gamma}$ :

$$
\mathcal{A}_{\gamma}=\left(l^{1}\left(\mathbb{Z}^{2 d}\right), \natural_{\gamma}, * \gamma\right) .
$$

Moreover, the obvious analogue of Young's inequality holds: if $\mathbf{a} \in l^{1}\left(\mathbb{Z}^{2 d}\right)$, the operator

$$
L_{\mathbf{a}}(\mathbf{b})=\mathbf{a} \natural_{\gamma} \mathbf{b}
$$

is bounded on every $l^{p}\left(\mathbb{Z}^{2 d}\right)$ with norm at most $\|\mathbf{a}\|_{1}$. The analogue of Wiener's theorem is as follows:

Theorem 3. If $\mathbf{a} \in \mathcal{A}_{\gamma}$, then the spectrum of the operator $L_{\mathbf{a}}$ as an operator on $l^{2}\left(\mathbb{Z}^{2 d}\right)$ is equal to its spectrum as an operator on $l^{1}\left(\mathbb{Z}^{2 d}\right)$. In particular, if $L_{\mathbf{a}}$ is invertible on $l^{2}\left(\mathbb{Z}^{2 d}\right)$, then a is invertible in the algebra $\mathcal{A}_{\gamma}$.

Section 4 is devoted to a sketch of the proofs of Theorems 2 and 3.

\section{2. von Neumann algebras generated by translations and modulations}

We review some notation and terminology. If $\mathcal{H}$ is a Hilbert space, $\mathcal{B}(\mathcal{H})$ denotes the algebra of bounded linear operators on $\mathcal{H}$. A von Neumann algebra on $\mathcal{H}$ is a weakly closed $*$-subalgebra of $\mathcal{B}(\mathcal{H})$. If $\mathcal{M} \subset \mathcal{B}(\mathcal{H})$, its commutant $\mathcal{M}^{\prime}$ is the von Neumann algebra of all $B \in \mathcal{B}(\mathcal{H})$ that commute with all $A \in \mathcal{N}$. A fundamental theorem of von Neumann states that if $\mathcal{M}$ is itself a von Neumann algebra, then $\left(\mathcal{M}^{\prime}\right)^{\prime}=\mathcal{M}$. Takesaki [22] is a good general reference for the von Neumann algebra theory needed here. Nelson [18] has given a particularly nice proof of the theorem just quoted.

Given $\alpha, \beta>0$, let $\mathcal{M}_{\alpha, \beta}$ be the von Neumann algebra on $L^{2}\left(\mathbb{R}^{d}\right)$ generated by the operators $M_{\beta j}$ and $T_{\alpha k}\left(j, k, \in \mathbb{Z}^{d}\right)$, that is, the von Neumann algebra generated by the group $G_{\alpha, \beta}$. The first fundamental fact about $\mathcal{M}_{\alpha, \beta}$ is the following. 


\section{PROPOSITION 1.}

$\mathcal{M}_{\alpha, \beta}^{\prime}=\mathcal{M}_{1 / \beta, 1 / \alpha}$

This is a special case of a theorem of Takesaki [21], of which there are several proofs in the literature (see [20]). It is obvious that $\mathcal{M}_{1 / \beta, 1 / \alpha} \subset \mathcal{M}_{\alpha, \beta}^{\prime}$ because $M_{\omega}$ commutes with $T_{x}$ precisely when $\omega \cdot x \in \mathbb{Z}$. A simple proof of the reverse inclusion can be found in Appendix 6.1 of [7]. Given $S \in \mathcal{M}_{\alpha, \beta}^{\prime}$ and $T \in \mathcal{M}_{1 / \beta, 1 / \alpha}^{\prime}$, one shows by obtaining explicit representations for $S$ and $T$ that $S T=T S$; hence $\mathcal{M}_{\alpha, \beta}^{\prime} \subset\left(\mathcal{M}_{1 / \beta, 1 / \alpha}^{\prime}\right)^{\prime}=\mathcal{M}_{1 / \beta, 1 / \alpha}$.

Next, we recall that if $\mathcal{A}$ is a $*$-subalgebra of $\mathcal{B}(\mathcal{H})$, a faithful trace on $\mathcal{A}$ is a linear functional $\tau$ on $\mathcal{A}$ such that $\tau(A B)=\tau(B A)$ for $A, B \in \mathcal{A}$ and $\tau\left(A^{*} A\right)>0$ for all nonzero $A \in \mathcal{A}$. If $\tau(I)=1, \tau$ is said to be normalized.

Let $\mathcal{N}_{\alpha, \beta}^{0}$ be the finite linear span of the operators $M_{\alpha j} T_{\beta k}\left(j, k \in \mathbb{Z}^{d}\right)$, and define $\tau$ on $\mathcal{M}_{\alpha, \beta}^{0}$ by

$$
\tau\left(\sum c_{j k} M_{\alpha j} T_{\beta k}\right)=c_{00}
$$

It is easily verified that $\tau$ is a normalized faithful trace on $\mathcal{M}_{\alpha, \beta}^{0}$. Moreover, suppose $R_{1}, \ldots, R_{N}$ are rectangular solids (products of intervals) in $\mathbb{R}^{d}$ whose interiors are disjoint, whose side lengths are all at $\operatorname{most} \min (1 / \alpha, \beta)$, and whose union is the cube $[0,1 / \alpha]^{d}$. If $\chi_{n}$ is the characteristic function of $R_{n}$, then

$$
\tau(A)=\alpha^{d} \sum_{1}^{N}\left\langle A \chi_{n}, \chi_{n}\right\rangle, \quad A \in \mathcal{M}_{\alpha, \beta}^{0} .
$$

Indeed, it suffices to verify (15) when $A=M_{\alpha j} T_{\beta k}$. Since the side lengths of the $R_{n}$ are at most $\beta$, their translates by amounts $\beta k(k \neq 0)$ are disjoint, so $\left\langle M_{\alpha j} T_{\beta k} \chi_{n}, \chi_{n}\right\rangle=0$ unless $k=0$, in which case (since $\bigcup_{1}^{N} R_{j}=[0,1 / \alpha]^{d}$ )

$$
\alpha^{d} \sum\left\langle M_{\alpha j} \chi_{n}, \chi_{n}\right\rangle=\alpha^{d} \int_{[0,1 / \alpha]^{d}} \mathrm{e}^{2 \pi i \alpha j \cdot t} \mathrm{~d} t=\delta_{j 0} .
$$

Equation (15) shows that $\tau$ extends uniquely to a normalized faithful trace on $\mathcal{M}_{\alpha, \beta}$ that is continuous in the weak operator topology, so that $\mathcal{M}_{\alpha, \beta}$ is a finite von Neumann algebra.

We remark that if $\alpha \beta$ is rational, say $\alpha \beta=p / q$, then the center of $\mathcal{M}_{\alpha, \beta}$ (that is, $\left.\mathcal{M}_{\alpha, \beta} \cap \mathcal{M}_{1 / \beta, 1 / \alpha}\right)$ is large: it contains all operators $M_{\alpha q j} T_{\beta q k}$ with $j, k \in \mathbb{Z}^{d}$. However, if $\alpha \beta$ is irrational, then the center of $\mathcal{M}_{\alpha, \beta}$ is trivial; that is, $\mathcal{M}_{\alpha, \beta}$ is a factor. (The ideas used in [7] to prove Proposition 1, as sketched above, easily yield a proof of this.) Since $\mathcal{M}_{\alpha, \beta}$ has a faithful trace, it is actually a factor of type $\mathrm{II}_{1}$.

We need one further ingredient. Given $\phi \in L^{2}\left(\mathbb{R}^{d}\right)$, let $A_{\phi}$ be the map from $L^{2}\left(\mathbb{R}^{d}\right)$ to the space of functions on $\mathbb{Z}^{2 d}$ given by

$$
\left(A_{\phi} f\right)_{j k}=\left\langle f, M_{\alpha j} T_{\beta k} \phi\right\rangle .
$$

(We encountered such maps earlier in the discussion of frames.) If $A_{\phi}$ is bounded from $L^{2}\left(\mathbb{R}^{d}\right)$ to $l^{2}\left(\mathbb{Z}^{2 d}\right)$, its adjoint is given by

$$
A_{\phi}^{*} \mathbf{c}=\sum c_{j k} M_{\alpha j} T_{\beta k} \phi,
$$

where the series is unconditionally convergent. 
Suppose $\phi, \psi \in L^{2}\left(\mathbb{R}^{d}\right)$ and $A_{\phi}, A_{\psi}$ are bounded from $L^{2}$ to $l^{2}$. We can then consider the 'generalized frame operator' $S_{\psi, \phi}=A_{\psi}^{*} A_{\phi}$ :

$$
S_{\psi, \phi} f=\sum\left\langle f, M_{\alpha j} T_{\beta k} \phi\right\rangle M_{\alpha j} T_{\beta k} \psi .
$$

An easy calculation that we leave to the reader shows that $S_{\psi, \phi}$ commutes with every $M_{\alpha j}$ and $T_{\beta k}$, so by Proposition $1, S_{\psi, \phi} \in \mathcal{M}_{1 / \beta, 1 / \alpha}$. The explicit expansion of $S_{\psi, \phi}$ in terms of the operators $M_{j / \beta} T_{k / \alpha}$ is quite pretty; it is known as the Janssen representation:

$$
S_{\psi, \phi}=(\alpha \beta)^{-d} \sum_{j, k}\left\langle\psi, M_{j / \beta} T_{k / \alpha} \phi\right\rangle M_{j / \beta} T_{k / \alpha} .
$$

Actually, without additional hypotheses on $\psi$ and $\phi$ the convergence of the series on the right is questionable, but it is sufficient for $\psi$ and $\phi$ to belong to the Feichtinger algebra $M_{1}^{1}$ defined by (10) with $v \equiv 1$.

\section{PROPOSITION 2.}

If $\phi$ and $\psi$ are in $M_{1}^{1}$, then $A_{\phi}$ and $A_{\psi}$ are bounded from $L^{2}\left(\mathbb{R}^{d}\right)$ to $l^{2}\left(\mathbb{Z}^{2 d}\right)$. Moreover, the Janssen representation (18) for $S_{\psi, \phi}$ is valid, and the series on the right converges absolutely in the operator norm.

For the proof, see Theorem 7.2.1 and Proposition 12.1.11 of [14].

The algebra $\mathcal{M}_{1 / \beta, 1 / \alpha}$ has a normalized faithful trace just like $\mathcal{M}_{\alpha, \beta}$, which we denote by $\tau^{\prime}$. The formula for the trace of $S_{\psi, \phi}$ is very simple.

\section{PROPOSITION 3.}

If $A_{\phi}$ and $A_{\psi}$ are bounded from $L^{2}\left(\mathbb{R}^{d}\right)$ to $l^{2}\left(\mathbb{Z}^{2 d}\right)$, then

$$
\tau^{\prime}\left(S_{\psi, \phi}\right)=(\alpha \beta)^{-d}\langle\psi, \phi\rangle .
$$

This is an immediate corollary of (14) (with $1 / \beta, 1 / \alpha$ in place of $\alpha, \beta$ ) and the Janssen representation if $\phi, \psi \in M_{1}^{1}$. The general case is not hard to prove from formula (15) (see [7]).

\section{Time-frequency density of complete Gabor systems}

We recall that if $\mathcal{M}$ is a von Neumann algebra on $\mathcal{H}$, a vector $v \in \mathcal{H}$ is called cyclic for $\mathcal{M}$ if $\{A v: A \in \mathcal{M}\}$ is dense in $\mathcal{H}$, and $\mathcal{M}$ is called cyclic if it has a cyclic vector. The Gabor system $\mathcal{G}(\phi, \alpha, \beta)$ spans $L^{2}\left(\mathbb{R}^{d}\right)$ precisely when $\phi$ is a cyclic vector for $\mathcal{M}_{\alpha, \beta}$, so Theorem 1 can be restated as follows:

$$
\text { If } \mathcal{M}_{\alpha, \beta} \text { is cyclic, then } \alpha \beta \leq 1 .
$$

The first proof of Theorem 1, in a sense, appeared before the question was even posed. That is, 19 is a consequence of a theorem of Rieffel [20] concerning the 'coupling function' for (a generalization of) $\mathcal{M}_{\alpha, \beta}$ and its commutant. The connection with Rieffel's theorem was first pointed out in Daubechies [4]. However, Rieffel's paper [20] is quite technical, and for those who are not specialists in operator algebras (including the present 
writer) the arguments in it, and the notion of 'coupling function' itself, are hard to grasp. We shall not attempt to describe them further.

At about the same time as [4], another proof of Theorem 1, for the case $d=1$, was given by Baggett [1]. Baggett's argument is of interest because it explicitly develops the connection of the problem with the representation theory of the discrete Heisenberg group $\mathbb{H}_{1}$. (It can be generalized to the $d$-dimensional case.)

We recall that the group $G_{\alpha, \beta}$ generated by the operators $M_{\alpha j}$ and $T_{\beta k}$ is the image of $\mathbb{H}_{1}$ under the unitary representation $\pi_{\alpha, \beta}$ defined by (7). Baggett relates $\pi_{\alpha, \beta}$ to some other representations of $\mathbb{H}_{1}$. First, let $H$ be the Abelian subgroup of $\mathbb{H}_{1}$ consisting of elements of the form $(j, 0, l)(j, l \in \mathbb{Z})$, and for $\gamma, \delta \in \mathbb{R}$ let $\sigma_{\gamma, \delta}$ be the representation of $\mathbb{H}_{1}$ induced from the character $\chi_{\gamma, \delta}(j, 0, l)=\mathrm{e}^{2 \pi i(\delta j-\gamma l)}$ of $H . \sigma_{\gamma, \delta}$ acts on $l^{2}(\mathbb{Z})$ by

$$
\left[\sigma_{\gamma, \delta}(j, k, l) \mathbf{c}\right]_{n}=\mathrm{e}^{2 \pi i(\delta j-\gamma l+n \gamma j)} c_{n-k}
$$

Next, for $\eta>0$ let $\Sigma_{\gamma, \eta}$ be the representation of $\mathbb{H}_{1}$ defined as the direct integral

$$
\Sigma_{\gamma, \eta}=\int_{[0, \eta)}^{\oplus} \sigma_{\gamma, \delta} \mathrm{d} \delta,
$$

which acts on $L^{2}([0, \eta) \times \mathbb{Z})$ in the obvious way, and let $\mathcal{N}_{\gamma, \eta}$ be the von Neumann algebra on $L^{2}([0, \eta) \times \mathbb{Z})$ generated by the operators $\Sigma_{\gamma, \eta}(h), h \in \mathbb{H}_{1}$. Baggett establishes the following facts:

(i) $\pi_{\alpha, \beta}$ is unitarily equivalent to $\Sigma_{\gamma, \gamma}$ where $\gamma=\alpha \beta$.

(ii) $\Sigma_{\gamma, \eta}$ is unitarily equivalent to $\Sigma_{\gamma^{\prime}, \eta^{\prime}}$ if and only if $\gamma=\gamma^{\prime}$ and $\eta=\eta^{\prime}$.

(iii) $\mathcal{N}_{\gamma, \eta}$ is isomorphic to $\mathcal{N}_{\gamma, \gamma}$ whenever $\eta \geq \gamma$.

(iv) $\Sigma_{\gamma, 1}$ is unitarily equivalent to the representation of $\mathbb{H}_{1}$ induced from the central character $\chi_{\gamma}(0,0, l)=\mathrm{e}^{-2 \pi i \gamma l}$.

The proof of Theorem 1, in the form 19, now proceeds by contradiction. Indeed, suppose that $\gamma=\alpha \beta>1$ but $\mathcal{M}_{\alpha, \beta}$ is cyclic. Then $\mathcal{M}_{\alpha, \beta}^{\prime}=\mathcal{M}_{1 / \beta, 1 / \alpha}$ is also cyclic since $1 / \alpha \beta<1$. (If $1 / \alpha \beta<1$, it is easy to construct $\phi \in L^{2}\left(\mathbb{R}^{d}\right)$ such that $\mathcal{G}(\phi, 1 / \beta, 1 / \alpha)$ is a frame for $L^{2}\left(\mathbb{R}^{d}\right)$; see [6].) Hence, by (i), $\mathcal{N}_{\gamma, \gamma}$ and its commutant are both cyclic. On the other hand, from (iv) and the theory of induced representations, $\mathcal{N}_{\gamma, 1}$ and its commutant are also both cyclic. But then, by well-known facts about von Neumann algebras that can be found in [22], the fact (iii) that $\mathcal{N}_{\gamma, \gamma}$ and $\mathcal{N}_{\gamma, 1}$ are isomorphic implies that they are actually unitarily equivalent, which contradicts (ii).

A few years after Daubechies [4] and Baggett [1], Daubechies et al [7] found another proof of Theorem 1 that uses the trace $\tau^{\prime}$ on $\mathcal{M}_{1 / \beta, 1 / \alpha}$ and the operators $A_{\phi}$ and $S_{\psi, \phi}$ defined by (16) and (17) in a very efficient way. (Like [1], this paper deals explicitly only with the case $d=1$, but the generalization to arbitrary $d$ is entirely straightforward.)

Suppose that $\psi \in L^{2}\left(\mathbb{R}^{d}\right)$ is a cyclic vector for $\mathcal{M}_{\alpha, \beta}$. If $A_{\psi}$ is not bounded from $L^{2}\left(\mathbb{R}^{d}\right)$ to $l^{2}\left(\mathbb{Z}^{2 d}\right)$, we regard it as an unbounded linear map with domain $D\left(A_{\psi}\right)=\{f \in$ $\left.L^{2}: \sum\left|\left\langle f, M_{\alpha j} T_{\beta k} \psi\right\rangle\right|^{2}<\infty\right\}$; as such, it is always closed and densely defined. It follows, by a theorem of von Neumann, that $S_{\psi, \psi}=A_{\psi}^{*} A_{\psi}$ is a positive self-adjoint operator on $L^{2}\left(\mathbb{R}^{d}\right)$. As in the bounded case, it commutes with the $M_{\alpha j}$ and $T_{\beta k}$, and so does $\left(\varepsilon I+S_{\psi, \psi}\right)^{-1}$ for any $\varepsilon>0$. It follows that if we set

$$
\phi=\left(\varepsilon I+S_{\psi, \psi}\right)^{-1} \psi
$$


then for any $f \in L^{2}\left(\mathbb{R}^{d}\right)$,

$$
\begin{aligned}
\left(A_{\phi} f\right)_{j k} & =\left\langle f, M_{\alpha j} T_{\beta k}\left(\varepsilon I+S_{\psi, \psi}\right)^{-1} \psi\right\rangle \\
& =\left\langle\left(\varepsilon I+S_{\psi, \psi}\right)^{-1} f, M_{\alpha j} T_{\beta k} \psi\right\rangle=\left(A_{\psi}\left(\varepsilon I+S_{\psi, \psi}\right)^{-1} f\right)_{j k},
\end{aligned}
$$

that is,

$$
A_{\phi}=A_{\psi}\left(\varepsilon I+S_{\psi, \psi}\right)^{-1}
$$

and hence

$$
S_{\psi, \psi}\left(\varepsilon I+S_{\psi, \psi}\right)^{-1}=A_{\psi}^{*} A_{\phi} .
$$

Next, Daubechies et al [7] showed that the formula in Proposition 3 for the trace of $S_{\psi, \phi}=$ $A_{\psi}^{*} A_{\phi}$ remains valid provided only $S_{\psi, \phi}$ is a bounded operator, even if $A_{\psi}$ or $A_{\phi}$ is not. By 20], that is the case for the $\psi$ and $\phi$ under consideration here, so

$$
(\alpha \beta)^{d} \tau^{\prime}\left(S_{\psi, \psi}\left(\varepsilon I+S_{\psi, \psi}\right)^{-1}\right)=(\alpha \beta)^{d} \tau^{\prime}\left(A_{\psi}^{*} A_{\phi}\right)=\langle\psi, \phi\rangle .
$$

Now, by the spectral functional calculus, $S_{\psi, \psi}\left(\varepsilon I+S_{\psi, \psi}\right)^{-1}$ converges strongly as $\varepsilon \rightarrow 0$ to the orthogonal projection onto the closure of the range of $S_{\psi, \psi}$. But since $\psi$ is cyclic for $\mathcal{M}_{\alpha, \beta}$, this range is dense in $L^{2}$, so $S_{\psi, \psi}\left(\varepsilon I+S_{\psi, \psi}\right)^{-1}$ converges strongly to $I$. The formula analogous to 15 for $\tau^{\prime}$ shows that $\tau^{\prime}$ is continuous with respect to strong convergence, so

$$
(\alpha \beta)^{d}=(\alpha \beta)^{d} \tau^{\prime}(I)=\langle\psi, \phi\rangle .
$$

On the other hand, we have

$$
\langle\psi, \phi\rangle=\left\langle\left(\varepsilon I+A_{\psi}^{*} A_{\psi}\right) \phi, \phi\right\rangle=\varepsilon\|\phi\|^{2}+\left\|A_{\psi} \phi\right\|^{2} \geq\left\|A_{\psi} \phi\right\|^{2},
$$

and if $\mathbf{e} \in l^{2}\left(\mathbb{Z}^{2 d}\right)$ is defined by $e_{j k}=\delta_{j 0} \delta_{k 0}$,

$$
\langle\psi, \phi\rangle=\left\langle A_{\psi}^{*} \mathbf{e}, \phi\right\rangle=\left\langle\mathbf{e}, A_{\psi} \phi\right\rangle \leq\left\|A_{\psi} \phi\right\| .
$$

(No absolute values are needed since $\langle\psi, \phi\rangle=(\alpha \beta)^{d}>0$.) These two inequalities imply that $\langle\psi, \phi\rangle^{2} \leq\langle\psi, \phi\rangle$ and hence $(\alpha \beta)^{d}=\langle\psi, \phi\rangle \leq 1$. Thus the proof is complete.

At about the same time as Daubechies et al [7], Ramanathan and Steger [19] found a more elementary argument to prove, and indeed generalize, Theorem 1 . They avoid von Neumann algebras but use an idea from a different branch of harmonic analysis: the notion of asymptotic density of a discrete set first exploited by Beurling in his work on balayage for the Fourier transform [3].

To wit, let $\Lambda$ be a discrete subset of $\mathbb{R}^{2 d}=\mathbb{R}^{d} \times \mathbb{R}^{d}$, and for $\phi \in L^{2}\left(\mathbb{R}^{d}\right)$, let $\mathcal{G}(\phi, \Lambda)$ be the corresponding Gabor system:

$$
\mathcal{G}(\phi, \Lambda)=\left\{M_{\omega} T_{x} \phi:(\omega, x) \in \Lambda\right\} .
$$

We are interested in the question of whether $\mathcal{G}(\phi, \Lambda)$ spans $L^{2}$. For $r>0$ and $(\eta, a) \in \mathbb{R}^{2 d}$, let $B_{r}(\eta, a)$ be the ball of radius $r$ about $(\eta, a)$, and let $v(r)=\left(2 \pi^{d} / d !\right) r^{2 d}$ be its volume. Define

$$
v^{-}(r)=\min _{(\eta, a) \in \mathbb{R}^{2 d}} \operatorname{card}\left(\Lambda \cap B_{r}(\eta, a)\right)
$$


and define the lower density of $\Lambda$ to be

$$
D^{-}(\Lambda)=\liminf _{r \rightarrow \infty} \frac{v^{-}(r)}{v(r)} .
$$

(There is a corresponding notion of upper density, but we shall not need it.)

We shall say that $\mathcal{G}(\phi, \Lambda)$ has the homogeneous approximation property if for every $f \in$ $L^{2}\left(\mathbb{R}^{d}\right)$ and $\varepsilon>0$ there is an $R>0$ such that for every $(\eta, a) \in \mathbb{R}^{2 d}$ there is a finite linear combination $h$ of the $M_{\omega} T_{x} \phi$ 's with $(\omega, x) \in \Lambda \cap B_{R}(\eta, a)$ such that $\left\|h-M_{\eta} T_{a} f\right\|_{2}<\varepsilon$. (This definition is quite a mouthful. The homogeneous approximation property implies that $\mathcal{G}(\phi, \Lambda)$ spans $L^{2}\left(\mathbb{R}^{d}\right)$, as one sees simply by taking $(\eta, a)=(0,0)$. But it is stronger: it means not only that each $f \in L^{2}$ can be approximated by finite linear combinations of $M_{\omega} T_{x} \phi$ 's with $(\omega, x) \in \Lambda$, but that translates and modulates of $f$ by arbitrary amounts $(\eta, a)$ can be uniformly approximated by linear combinations of $M_{\omega} T_{x} \phi$ 's with $(\omega, x) \in \Lambda$ not too far from $(\eta, a)$.)

The main result of Ramanathan and Steger [19] is the following:

Theorem 4. If $\mathcal{G}(\phi, \Lambda)$ has the homogeneous approximation property, then $D^{-}(\Lambda) \geq 1$.

Let $\chi$ be the characteristic function of the unit cube. The idea of the proof is to compare $\mathcal{G}(\phi, \Lambda)$ with $\mathcal{G}\left(\chi, \mathbb{Z}^{2 d}\right)$, which is an orthonormal basis for $L^{2}\left(\mathbb{R}^{d}\right)$. For $(\eta, a) \in \mathbb{R}^{2 d}$ and $r>0$, let

$$
\begin{gathered}
V_{r}(\eta, a)=\text { linear span of }\left\{M_{j} T_{k} \chi:(j, k) \in \mathbb{Z}^{2 d} \cap B_{r}(\eta, a)\right\}, \\
W_{r}(\eta, a)=\text { linear span of }\left\{M_{\omega} T_{x} \phi:(\omega, x) \in \Lambda \cap B_{r}(\eta, a)\right\} .
\end{gathered}
$$

Next, given $R>0$, let $T$ be the restriction to the finite-dimensional space $V_{r}(\eta, a)$ of the operator $P_{V_{r}(\eta, a)} \circ P_{W_{r+R}(\eta, a)}\left(P_{X}=\right.$ orthogonal projection onto $\left.X\right)$. Since $T$ is a composition of projections, its eigenvalues lie in $[0,1]$, so its trace is dominated by its rank. Thus,

$$
\operatorname{tr}(T) \leq \operatorname{dim} W_{r+R}(\eta, a)=\operatorname{card}\left(\Lambda \cap B_{r+R}(\eta, a)\right) .
$$

On the other hand, the homogeneous approximation property implies that for any $\varepsilon>0$ we can find $R>0$ such that $\left\|T\left(M_{j} T_{k} \chi\right)-M_{j} T_{k} \chi\right\|_{2}<\varepsilon$ for all $(j, k) \in \mathbb{Z}^{2 d} \cap B_{r}(\eta, a)$, and hence

$$
\operatorname{tr}(T)=\sum_{\mathbb{Z}^{2 d} \cap B_{r}(\eta, a)}\left\langle T M_{j} T_{k} \chi, M_{j} T_{k} \chi\right\rangle \geq(1-\varepsilon) \operatorname{card}\left(\mathbb{Z}^{2 d} \cap B_{r}(\eta, a)\right) .
$$

Therefore,

$$
(1-\varepsilon) \frac{\operatorname{card}\left(\mathbb{Z}^{2 d} \cap B_{r}(\eta, a)\right)}{v(r)} \leq \frac{\operatorname{card}\left(\Lambda \cap B_{r+R}(\eta, a)\right)}{v(r+R)} \frac{v(r+R)}{v(r)} .
$$

Pick sequences $r_{n} \rightarrow \infty$ and $\left(\eta_{n}, a_{n}\right) \in \mathbb{R}^{2 d}$ such that $\operatorname{card}\left(\Lambda \cap B_{r_{n}+R}\left(\eta_{n}, a_{n}\right)\right) / v\left(r_{n}+R\right) \rightarrow$ $D^{-}(\Lambda)$. We have $\operatorname{card}\left(\mathbb{Z}^{2 d} \cap B_{r_{n}}\left(\eta_{n}, a_{n}\right)\right) / v\left(r_{n}\right) \rightarrow 1$ and $v\left(r_{n}+R\right) / v\left(r_{n}\right)=\left(r_{n}+R\right)^{d} / r_{n}^{d} \rightarrow$ 1 , and hence

$$
1-\varepsilon \leq D^{-}(\Lambda) .
$$

Since $\varepsilon$ is arbitrary, the proof is complete. 
Ramanathan and Steger further show that if $\Lambda$ is a lattice and $\mathcal{G}(\phi, \Lambda)$ spans $L^{2}\left(\mathbb{R}^{d}\right)$, then $\mathcal{G}(\phi, \Lambda)$ has the homogeneous approximation property. (The proof is straightforward.) Since $D^{-}(\Lambda)=1 / \operatorname{vol}\left(\mathbb{R}^{2 d} / \Lambda\right)$ in this case, we have the following generalization of Theorem 1.

Theorem 5. If $\Lambda$ is a lattice in $\mathbb{R}^{2 d}$ such that $\operatorname{vol}\left(\mathbb{R}^{2 d} / \Lambda\right)>1$, there is no $\phi$ in $L^{2}\left(\mathbb{R}^{d}\right)$ such that $\mathcal{G}(\phi, \Lambda)$ spans $L^{2}\left(\mathbb{R}^{d}\right)$.

More recently, Gabardo and Han [12] have extended Theorem 1 to a very general setting using the same circle of ideas as Daubechies et al [7], but arranged rather differently. They define a group-like unitary system to be a countable collection $\mathcal{U}$ of unitary operators on a separable Hilbert space $\mathcal{H}$ with the property that the group $G(\mathcal{U})$ generated by $\mathcal{U}$ lies in $\{\lambda U: U \in \mathcal{U}, \lambda \in \mathbb{C}\}$. Thus, $\left\{M_{\alpha j} T_{\beta k}: j, k \in \mathbb{Z}^{d}\right\}$ is a group-like unitary system on $L^{2}\left(\mathbb{R}^{d}\right)$, and any projective representation of a countable group gives rise to a group-like unitary system.

Given a group-like unitary system $\mathcal{U}$ on $\mathcal{H}$ and $v \in \mathcal{H}$, one has the linear map $A_{v}$ from $\mathcal{H}$ to functions on $\mathcal{U}$ given by $\left(A_{v} w\right)_{U}=\langle w, U v\rangle$; Gabardo and Han assume that the set of $v \in \mathcal{H}$ such that $A_{v}$ is bounded from $\mathcal{H}$ to $l^{2}(\mathcal{U})$ is dense in $\mathcal{H}$. Under this condition, they show that $\mathcal{H}$ is the orthogonal direct sum of subspaces $\mathcal{H}_{i}(i \in I)$, each of which is $\mathcal{U}$-invariant, and each of which possesses a vector $v_{i}$ such that $\|w\|^{2}=\sum_{U \in \mathcal{U}}\left|\left\langle w, U v_{i}\right\rangle\right|^{2}$ for all $w \in \mathcal{H}_{i}$ (that is, $\left\{U v_{i}: U \in \mathcal{U}\right\}$ is a 'normalized tight frame' for $\mathcal{H}_{i}$ ). They show that $\sum_{i \in I}\left\|v_{i}\right\|^{2}$ depends only on $\mathcal{U}$, and they define the redundancy of $\mathcal{U}$ to be

$$
r(\mathcal{U})=\left(\sum\left\|v_{i}\right\|^{2}\right)^{-1} \text {. }
$$

They then show that:

(i) $\mathcal{U}$ has a cyclic vector (a vector $v \in \mathcal{H}$ such that the linear span of $\{U v: U \in \mathcal{U}\}$ is dense in $\mathcal{H}$ ) if and only if $r(\mathcal{U}) \geq 1$.

(ii) If the index set $I$ is finite, then the commutant $\mathcal{U}^{\prime}$ is a finite von Neumann algebra. The formula $\tilde{\tau}\left(A_{w}^{*} A_{v}\right)=\langle w, v\rangle$ (for any $v$ and $w$ such that $A_{v}$ and $A_{w}$ are bounded from $\mathcal{H}$ to $l^{2}(\mathcal{U})$ ) determines a faithful trace on $\mathcal{U}^{\prime}$, and $r(\mathcal{U})=\tilde{\tau}(I)$.

In the case $\mathcal{U}=\left\{M_{\alpha j} T_{\beta k}: j, k \in \mathbb{Z}^{d}\right\}$, one can take the vectors $v_{i}$ to be the characteristic functions $\chi_{n}$ in 15 and the subspaces $\mathcal{H}_{i}$ to be the $\mathcal{U}$-invariant subspaces they generate. Then $\mathcal{U}^{\prime}=\mathcal{M}_{1 / \beta, 1 / \alpha}$, and by Proposition 3, Gabardo and Han's trace $\tilde{\tau}$ is $(\alpha \beta)^{d} \tau^{\prime}$, so their results (i) and (ii) imply Theorem 1. In fact, without much additional effort, they yield Theorem 5 for the case of lattices of the form $A \mathbb{Z}^{d} \times B \mathbb{Z}^{d}$ where $A, B \in G L(n, \mathbb{R})$.

Another generalization of Theorem 1 has been obtained by Bekka [2]. Bekka considers an irreducible square-integrable representation $\pi$ of a unimodular locally compact group $G$ on a separable Hilbert space $\mathcal{H}$, and a discrete subgroup $\Gamma$ of $G$ such that the volume of $G / \Gamma$ is finite. He proves results relating (i) the formal dimension of $\pi$ (i.e., the constant $d_{\pi}$ such that $\|\xi\|^{2}\|\eta\|^{2}=d_{\pi} \int_{G}|\langle\xi, \pi(g) \eta\rangle|^{2} d g$ ), (ii) the volume of $G / \Gamma$, and (iii) the 'center-valued von Neumann dimension' of $\mathcal{H}, \operatorname{cdim}(\mathcal{H})$, as a $\mathrm{VN}(\Gamma)$-module, where $\mathrm{VN}(\Gamma)$ is the von Neumann algebra on $l^{2}(\Gamma)$ generated by the left regular representation. (We shall not attempt to describe $\operatorname{cdim}(\mathcal{H})$ other than to say that it is an element of the center of $\mathrm{VN}(\Gamma)$.) Bekka's abstract version of Theorem 1 in this setting is as follows:

Theorem 6. If there is a vector $v \in \mathcal{H}$ such that $\{\pi(g) v: g \in \Gamma\}$ spans $\mathcal{H}$, then $\operatorname{cdim}(\mathcal{H}) \leq I$. 
When specialized to the case where $G$ is the (reduced) real Heisenberg group and $\Gamma$ is the discrete Heisenberg group, this yields Theorem 5. (Note that Bekka normalizes his frequency variables differently, with the result that some $2 \pi$ 's appear in his formulas that are not in ours. But in fact each of his $2 \pi$ 's should be $(2 \pi)^{d}$.)

\section{Wiener's theorem and Gabor frames}

We now turn to the proofs of Theorems 2 and 3, following the arguments of Gröchenig and Leinert [15]. We begin with Theorem 3 and then obtain Theorem 2 from it.

Recall that we are concerned with the Banach $*$-algebra $\mathcal{A}_{\gamma}$, which is $l^{1}\left(\mathbb{Z}^{2 d}\right)$ equipped with the product and involution defined by 11 and (12), and that for $\mathbf{a} \in \mathcal{A}_{\gamma}, L_{\mathbf{a}}$ denotes the operator $L_{\mathbf{a}}(\mathbf{b})=\mathbf{a} \bigsqcup_{\gamma} \mathbf{b}$. If $X$ is a space of functions on $\mathbb{Z}^{2 d}$ on which $L_{\mathbf{a}}$ is a bounded operator, we denote the spectrum and spectral radius of $L_{\mathbf{a}}$ on $X$ by $\sigma_{X}(\mathbf{a})$ and $\rho_{X}(\mathbf{a})$, respectively. That is,

$$
\begin{aligned}
& \sigma_{X}(\mathbf{a})=\left\{\lambda \in \mathbb{C}: \lambda I-L_{\mathbf{a}} \text { is not invertible on } X\right\}, \\
& \rho_{X}(\mathbf{a})=\sup \left\{|\lambda|: \lambda \in \sigma_{X}(\mathbf{a})\right\}=\lim _{n \rightarrow \infty}\left\|L_{\mathbf{a}}^{n}\right\|_{\mathcal{B}(X)}^{1 / n} .
\end{aligned}
$$

Here and in the sequel, $\mathcal{B}(X)$ denotes the space of bounded linear operators on $\mathcal{X}$. Moreover, we abbreviate $l^{p}\left(\mathbb{Z}^{2 d}\right)$ as $l^{p}$. Our goal is to prove that $\sigma_{l_{1}}(\mathbf{a})=\sigma_{l^{2}}(\mathbf{a})$ for all $\mathbf{a} \in l^{1}=$ $\mathcal{A}_{\gamma}$.

Just as twisted convolution on $\mathbb{R}^{2 d}$ is closely related to ordinary convolution on the reduced Heisenberg group (the real Heisenberg group modified so that its center is a circle rather than a line; see pp. 25-26 of [10]), so the twisted convolution $\natural_{\gamma}$ on $\mathbb{Z}^{2 d}$ is closely related to ordinary convolution on a modification of the discrete Heisenberg group $\mathbb{H}_{d}$, namely, the group $H_{\gamma}$ whose underlying set is $\mathbb{Z}^{d} \times \mathbb{Z}^{d} \times \mathbb{T}$ ( $\mathbb{T}=$ the group of complex numbers of modulus one) and whose group law is

$$
(j, k, \zeta)\left(j^{\prime}, k^{\prime}, \zeta^{\prime}\right)=\left(j+j^{\prime}, k+k^{\prime}, \zeta \zeta^{\prime} \mathrm{e}^{-2 \pi i \gamma k \cdot j^{\prime}}\right)
$$

(Note that $(j, k, l) \mapsto\left(j, k, \mathrm{e}^{-2 \pi i \gamma l}\right)$ is a homomorphism from $\mathbb{H}_{d}$ to $H_{\gamma}$. It is injective if $\gamma$ is irrational, and its kernel is $q Z$ as in 8 if $\gamma=p / q$ in lowest terms.) Indeed, $L^{1}\left(H_{\gamma}\right)$ is a Banach $*$-algebra under convolution and the usual involution $f^{*}(\xi)=\overline{f\left(\xi^{-1}\right)}$, and the map $J: \mathcal{A}_{\gamma} \rightarrow L^{1}\left(H_{\gamma}\right)$ defined by

$$
J(\mathbf{a})(j, k, \zeta)=\zeta^{-1} a_{j k}
$$

is easily seen to be a $*$-isomorphism of $\mathcal{A}_{\gamma}$ onto the subalgebra of $L^{1}\left(H_{\gamma}\right)$ consisting of those functions $f$ that satisfy $f(j, k, \zeta)=\zeta^{-1} f(j, k, 1)$.

With this in mind, the ingredients from abstract harmonic analysis that are needed to prove Theorem 2 are as follows. In them we employ an obvious modification of the notation introduced earlier: if $f$ is an element of the Banach algebra $\mathcal{A}, \sigma_{\mathcal{A}}(f)$ and $\rho_{\mathcal{A}}(f)$ denote its spectrum and spectral radius. The first two lemmas are theorems of Hulanicki [16] and Ludwig [17], respectively.

Lemma 1.[16]. Suppose $\mathcal{S}$ is a $*$-subalgebra of a Banach $*$-algebra $\mathcal{A}$, and there exists a faithful *-representation of $\mathcal{A}$ on a Hilbert space $\mathcal{H}$ such that if $f \in \mathcal{S}$ and $f=f^{*}$ then $\|\pi(f)\|_{\mathcal{B}(\mathcal{H})}=\rho_{\mathcal{A}}(f)$. Then for any $f \in \mathcal{S}$ with $f=f^{*}$ we have $\sigma_{\mathcal{A}}(f)=\sigma_{\mathcal{B}(\mathcal{H})}(\pi(f))$. 
Recall that a Banach $*$-algebra $\mathcal{A}$ is called symmetric if $\sigma_{\mathcal{A}}(f) \subset[0, \infty)$ for all $f \in \mathcal{A}$ such that $f=f^{*}$.

Lemma 2.[17]. If $G$ is a locally compact nilpotent group, then $L^{1}(G)$ is symmetric.

Using the fact that every locally compact nilpotent group is amenable, some standard facts about amenable groups, and Lemmas 1 and 2, it is not hard to deduce the next result (Theorem 2.8 of [15]).

Lemma 3. Suppose $G$ is a locally compact nilpotent group and $f \in L^{1}(G)$ satisfies $f=f^{*}$. Then the convolution operator $C_{f}(g)=f * g$ satisfies $\sigma_{\mathcal{B}\left(L^{1}(G)\right)}\left(C_{f}\right)=\sigma_{\mathcal{B}\left(L^{2}(G)\right)}\left(C_{f}\right)$.

We can now sketch the proof of Theorem 3. Suppose $\mathbf{a} \in \mathcal{A}_{\gamma}$. If $\mathbf{a}=\mathbf{a}^{* \gamma}$, we employ the map $J$ defined by (21) to transfer the problem to $L^{1}\left(H_{\gamma}\right)$. Since $H_{\gamma}$ is nilpotent, the conclusion $\sigma_{l^{1}}(\mathbf{a})=\sigma_{l^{2}}(\mathbf{a})$ follows easily from Lemma 3 . The result for general $\mathbf{a}$ is now obtained by the following simple device. If $L_{\mathbf{a}}$ is invertible on $l^{2}$, then so is $L_{\mathbf{b}}$ where $\mathbf{b}=$ $a^{*} \natural_{\gamma} \mathbf{a}$ or $\mathbf{b}=\mathbf{a} \natural_{\gamma} \mathbf{a}^{* \gamma}$, and these b's satisfy $\mathbf{b}=\mathbf{b}^{* \gamma}$, so $\mathbf{a}^{* \gamma} \natural_{\gamma} \mathbf{a}$ and $\mathbf{a} \natural_{\gamma} \mathbf{a}^{* \gamma}$ are invertible in $l^{1}$ by the result just proved. But then $\left(\mathbf{a}^{*} \natural_{\gamma} \mathbf{a}\right)^{-1} \natural_{\gamma} \mathbf{a}^{* \gamma}$ and $\mathbf{a}^{*} \natural_{\gamma}\left(\mathbf{a} \natural_{\gamma} \mathbf{a}^{* \gamma}\right)^{-1}$ are left and right inverses for $\mathbf{a}$, respectively, so $\mathbf{a}$ is invertible in $l^{1}$. The desired conclusion $\sigma_{l^{1}}(\mathbf{a})=$ $\sigma_{l^{2}}(\mathbf{a})$ follows by applying this result to $\lambda \mathbf{e}-\mathbf{a}$ where $\mathbf{e}$ is the identity in the algebra $\mathcal{A}_{\gamma}=l^{1}\left(e_{j k}=\delta_{j 0} \delta_{k 0}\right)$.

Finally, we show how Gröchenig and Leinert [15] deduce Theorem 2 from Theorem 3. Let us suppose that $\mathcal{G}(\phi, \alpha, \beta)$ is a frame for $L^{2}\left(\mathbb{R}^{d}\right)$ and $\phi \in M_{v}^{1}$ where $v$ is a subexponential weight function; we wish to show that the frame operator $S=S_{\phi, \phi}$ (notation as in (17)) is invertible on $M_{v}^{1}$. The link with Theorem 3 comes through the following considerations.

Lemma 4. If $\mathbf{a} \in l^{1}\left(\mathbb{Z}^{2 d}\right)$, let

$$
\pi(\mathbf{a})=\sum a_{j k} M_{j / \beta} T_{k / \alpha} .
$$

Then $\pi$ is a $*$-representation of the algebra $\mathcal{A}_{\gamma}$ where $\gamma=1 / \alpha \beta$.

Let

$$
\tilde{v}(j, k)=v(j / \beta, k / \alpha), \quad j, k \in \mathbb{Z}^{d}
$$

and let

$$
l_{\tilde{v}}^{1}=\left\{\mathbf{a} \in l^{1}\left(\mathbb{Z}^{2 d}\right): \sum\left|a_{j k}\right| \tilde{v}(j, k)<\infty\right\} .
$$

Since $v$ is submultiplicative, it is easily verified that $l_{\tilde{v}}^{1}$ is a $*$-subalgebra of the twisted convolution algebra $\mathcal{A}_{\gamma}$, for any $\gamma>0$.

Lemma 5. If $\mathbf{a} \in l_{\tilde{v}}^{1}$, then $\pi(\mathbf{a})$ is bounded on $M_{v}^{1}$.

Lemma 6. If $\mathbf{a} \in l_{\tilde{v}}^{1}$, then $\rho_{l_{\tilde{v}}^{1}}(\mathbf{a})=\rho_{l^{1}}(\mathbf{a})$.

The proofs of Lemmas 4, 5, and 6 are all quite easy. The next one is a little deeper.

Lemma 7. If $\mathbf{a} \in l^{1}\left(\mathbb{Z}^{2 d}\right)$, then $\|\pi(\mathbf{a})\|_{\mathcal{B}\left(L^{2}\left(\mathbb{R}^{d}\right)\right)}=\left\|L_{\mathbf{a}}\right\|_{\mathcal{B}\left(l^{2}\left(\mathbb{Z}^{2 d}\right)\right)}$. 
The idea of the proof is as follows. Let $C^{*}\left(l^{1}\right)$ be the $C^{*}$ subalgebra of $\mathcal{B}\left(l^{2}\left(\mathbb{Z}^{2 d}\right)\right)$ generated by the operators $L_{\mathbf{a}}, \mathbf{a} \in l^{1}$, and let $C^{*}(\alpha, \beta)$ be the $C^{*}$ subalgebra of $\mathcal{B}\left(L^{2}\left(\mathbb{R}^{d}\right)\right)$ generated by the $M_{j / \beta} T_{k / \alpha}, j, k \in \mathbb{Z}^{d}$. One shows that the correspondence $L_{\mathbf{a}} \mapsto \pi(\mathbf{a})$ extends to an injective $*$-homomorphism from $C^{*}\left(l^{1}\right)$ to $C^{*}(\alpha, \beta)$. But injective *homomorphisms of $C^{*}$ algebras are always isometries.

Lemma 8. Suppose $\alpha, \beta>0$. If $\mathbf{a} \in L_{\tilde{v}}^{1}$ and $\pi(\mathbf{a})$ is invertible on $L^{2}\left(\mathbb{R}^{d}\right)$, then $\mathbf{a}$ is invertible in the algebra $l_{\tilde{v}}^{1} \subset \mathcal{A}_{\gamma}$, where $\gamma=1 / \alpha \beta$.

To prove this, first suppose that $\mathbf{a}=\mathbf{a}^{* \gamma}$. Then

$$
\|\pi(\mathbf{a})\|_{\mathcal{B}\left(L^{2}\right)}=\left\|L_{\mathbf{a}}\right\|_{\mathcal{B}\left(l^{2}\right)}=\rho_{l^{2}}(\mathbf{a})=\rho_{l^{1}}(\mathbf{a})=\rho_{l_{\bar{v}}^{1}}(\mathbf{a}),
$$

where the equalities are justified by Lemma 7 , the fact that $\mathbf{a}=\mathbf{a}^{* \gamma}$, Theorem 3 , and Lemma 6, respectively. Thus, we can apply Hulanicki's theorem (Lemma 1) with $\mathcal{S}=l_{\tilde{v}}^{1}$, $\mathcal{A}=\mathcal{A}_{\gamma}, \pi$ as in Lemma 4 , and $\mathcal{H}=L^{2}\left(\mathbb{R}^{d}\right)$ to conclude that $\sigma_{l_{\tilde{v}}}(\mathbf{a})=\sigma_{\mathcal{B}\left(L^{2}\right)}(\pi(\mathbf{a}))$. Since $0 \notin \sigma_{\mathcal{B}\left(L^{2}\right)}(\pi(\mathbf{a}))$ by assumption, $\mathbf{a}$ is invertible in $l_{\tilde{v}}^{1}$. The case $\mathbf{a} \neq \mathbf{a}^{* \gamma}$ now follows as in the proof of Theorem 3 .

At last we are ready to prove Theorem 2. Recall the Janssen representation (18, which is valid for the frame operator $S_{\phi, \phi}$ under consideration by Proposition 2. In terms of the notation of Lemma 4 , it says that

$$
S_{\phi, \phi}=\pi(\mathbf{b}), \quad \text { where } b_{j k}=\left\langle\phi, M_{j / \beta} T_{k / \alpha} \phi\right\rangle .
$$

Since $\phi \in M_{v}^{1}$, it is easy to verify that $\mathbf{b} \in l_{\tilde{v}}^{1}$, and since $\mathcal{G}(\phi, \alpha, \beta)$ is a frame for $L^{2}$, $S_{\phi, \phi}=\pi(\mathbf{b})$ is invertible on $L^{2}$. Hence $\mathbf{b}$ is invertible in $l_{\tilde{v}}^{1}$ by Lemma 8 , so $S_{\phi, \phi}^{-1}=\pi\left(\mathbf{b}^{-1}\right)$ is bounded on $M_{v}^{1}$ by Lemma 5 , and we are done.

\section{References}

[1] Baggett L, Processing a radar signal and representations of the discrete Heisenberg group, Colloq. Math. 60/61 (1990) 195-203

[2] Bekka B, Square integrable representations, von Neumann algebras and an application to Gabor analysis, J. Fourier Anal. Appl. 10 (2004) 325-349

[3] Beurling A, Balayage of Fourier-Stieltjes transforms, in Beurling's Collected Works, (Boston: Birkhäuser) (1989) vol. II, pp. 341-365

[4] Daubechies I, The wavelet transform, time-frequency localization and signal analysis, IEEE Trans. Inform. Theory 36 (1990) 961-1005

[5] Daubechies I, Ten lectures on wavelets (1992) (Philadelphia: Society for Industrial and Applied Mathematics)

[6] Daubechies I, Grossmann A and Meyer Y, Painless nonorthogonal expansions, Comm. Math. Phys. 27 (1986) 1271-1283

[7] Daubechies I, Landau H J and Landau Z, Gabor time-frequency lattices and the WexlerRaz identity, J. Fourier Anal. Appl. 1 (1995) 437-478

[8] Feichtinger H G and Gröchenig K, Gabor wavelets and the Heisenberg group: Gabor expansions and the short time Fourier transform from the group theoretical point of view, in Wavelets: A tutorial in theory and applications (ed.) C K Chui (1992) (Boston: Academic Press) pp. 359-397

[9] Feichtinger H G and Gröchenig K, Gabor frames and time-frequency analysis of distributions, J. Funct. Anal. 146 (1997) 464-495 
[10] Folland G B, Harmonic analysis in phase space (1989) (Princeton, NJ: Princeton Univ. Press)

[11] Folland G B, A course in abstract harmonic analysis (1995) (Boca Raton, Florida: CRC Press)

[12] Gabardo J-P and Han D, Frame representations for group-like unitary operator systems, J. Operator Theory 49 (2003) 223-244

[13] Gabor D, Theory of communication, J. Inst. Elec. Eng. 93(III) (1946) 429-457

[14] Gröchenig K, Foundations of time-frequency analysis (2001) (Boston: Birkhäuser)

[15] Gröchenig K and Leinert M, Wiener's lemma for twisted convolution and Gabor frames, J. Am. Math. Soc. 17 (2004) 1-17

[16] Hulanicki A, On the spectrum of convolution operators on groups with polynomial growth, Invent. Math. 17 (1972) 135-142

[17] Ludwig J, A class of symmetric and a class of Wiener group algebras, J. Funct. Anal. 31 (1979) 187-194

[18] Nelson E, Notes on non-commutative integration, J. Funct. Anal. 15 (1974) 103-116

[19] Ramanathan J and Steger T, Incompleteness of sparse coherent states, Appl. Comput. Harmon. Anal. 2 (1995) 148-153

[20] Rieffel M, Von Neumann algebras associated with pairs of lattices in Lie groups, Math. Ann. 257 (1981) 403-418

[21] Takesaki M, A generalized commutation theorem for the regular representation, Bull. Soc. Math. France 97 (1969) 289-297

[22] Takesaki M, Theory of Operator Algebras I (1979) (Berlin: Springer-Verlag) 